\title{
Rider motion identification during normal bicycling by means of principal component analysis
}

\author{
Jason K. Moore · J.D.G. Kooijman • A.L. Schwab • \\ Mont Hubbard
}

Received: 18 December 2009 / Accepted: 9 September 2010 / Published online: 9 October 2010

(C) The Author(s) 2010. This article is published with open access at Springerlink.com

\begin{abstract}
Recent observations of a bicyclist riding through town and on a treadmill show that the rider uses the upper body very little when performing normal maneuvers and that the bicyclist may, in fact, primarily use steering input for control. The observations also revealed that other motions such as lateral movement of the knees were used in low speed stabilization. In order to validate the hypothesis that there is little upper body motion during casual cycling, an in-depth motion capture analysis was performed on the bicycle and rider system.

We used motion capture technology to record the motion of three similar young adult male riders riding two different city bicycles on a treadmill. Each rider rode each bicycle while performing stability trials at speeds ranging from $2 \mathrm{~km} / \mathrm{h}$ to $30 \mathrm{~km} / \mathrm{h}$ : stabilizing while pedaling normally, stabilizing without pedaling, line tracking while pedaling, and stabilizing with no-hands. These tasks were chosen with the intent of examining differences in the kinematics at various speeds, the effects of pedaling on the system, upper body control motions and the differences in tracking and stabilization.

Principal component analysis was used to transform the data into a manageable set organized by the variance associated with the principal components. In this paper, these principal components were used to characterize various distinct kinematic motions that occur during
\end{abstract}

\author{
J.K. Moore $(\bowtie) \cdot$ M. Hubbard \\ Mechanical and Aerospace Engineering, University of California, Davis, One Shields Avenue, Davis, \\ CA 95616-5294, USA \\ e-mail: jkmoor@ucdavis.edu \\ M. Hubbard \\ e-mail: mhubbard@ucdavis.edu
}

J.D.G. Kooijman · A.L. Schwab

Laboratory for Engineering Mechanics, Delft University of Technology, Mekelweg 2, 2628 CD Delft, The Netherlands

J.D.G. Kooijman

e-mail: jodikooijman@gmail.com

A.L. Schwab
e-mail: a.1.schwab@tudelft.nl 
stabilization with and without pedaling. These motions were grouped on the basis of correlation and conclusions were drawn about which motions are candidates for stabilizationrelated control actions.

Keywords Bicycle $\cdot$ Principal component analysis · Motion capture $\cdot$ Human control

\section{Introduction}

Much progress has been made in understanding the rigid body dynamics of an uncontrolled bicycle $[1,2]$ and various control schemes have been explored for tracking purposes [3-5], but little is understood about how a bicyclist stabilizes a bicycle during normal riding. A bicycle and rider system is unique among vehicles in that the rider is 80 to $90 \%$ of the total mass of the system, the system is laterally unstable, and the rider is flexibly coupled to the bicycle in such a way that many body motions can be used as control inputs. Previous research into realistic bicycle control has focused on both steering and rider lean as control inputs, but there has been no experimental verification of which motions a rider actually uses for control. Recent observations of a bicyclist riding through town and on a treadmill [6] show that the rider moves the upper body very little when performing normal maneuvers and that the bicyclist may, in fact, primarily use steering input for control. This corresponds well with the fact that control by leaning requires high gains compared to the gains required for steering when employing an optimal control strategy on a model [3-5]. The observations also revealed that the rider may use other control inputs such as drastic knee movements at low speeds. These conclusions were drawn by visually reviewing video data, so a more rigorous objective method of characterizing the dominant movements of the bicyclist while stabilizing a bicycle was needed. In order to validate the hypothesis that there is little upper body motion during normal cycling, motion capture techniques were used on the bicycle and rider system with the intent to employ principal component analysis to identify the major motion patterns.

Principal component analysis has successfully been used with data collected from motion capture techniques to identify the dominant modes of motion of a person walking on a treadmill [7] and to characterize different types of walking. We use similar methods for steady, normal bicycle riding on a treadmill. Cyclic motions, such as pedaling, are easily identified and separated from the other less cyclic control actions. Identifying the patterns of movement gives insight into which body movements are primarily used and are candidates for control inputs. This will be valuable for our overall research goals that include the design of a realistic biomechanical-based control system of a bicycle rider, among other things.

\section{Experiments}

To test our hypotheses, three riders performed a set of stability tasks in a controlled environment while the motion of the bicycle and rider were collected with a motion capture system. The tasks were performed on a $3 \times 5$ meter treadmill Fig. 1 capable of belt speeds up to $35 \mathrm{~km} / \mathrm{h}$. The treadmill was chosen because the envelope of space was suitable for the motion capture system and it eliminated any disturbances such as wind, rough ground, and obstacles. We chose three male riders of similar age [31, 23, 26 years], build [height $(1.76,1.84,1.83 \mathrm{~m})$ and mass $(72,74,72 \mathrm{~kg})]$. We also used two different Dutch bicycles: a 2008 Batavus Browser with a 3 speed hub and a 2008 Batavus Stratos Deluxe with 


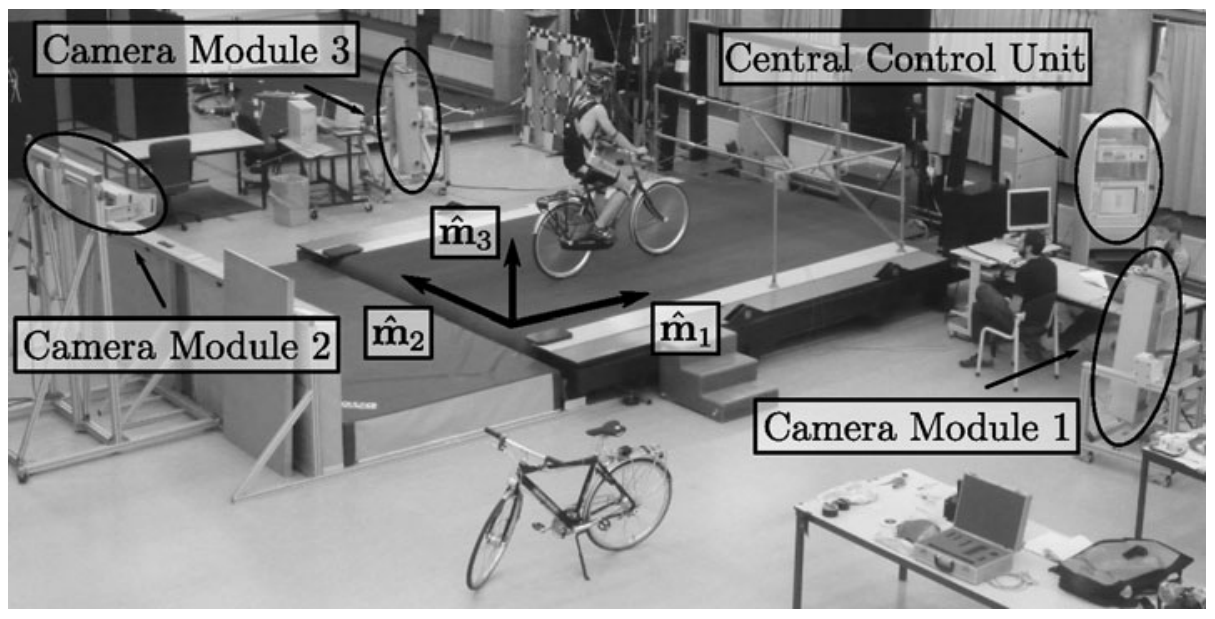

Fig. 1 The $3 \times 5 \mathrm{~m}$ treadmill at the Vrije Universiteit Amsterdam

a 7 speed hub. The Browser is described by the manufacturer as "stable" and the Stratos Deluxe as "nervous."

We made use of the Optotrak Certus Motion Capture System [8] to record the motion of the bicycle and rider during the stability tasks. The system is based on active infrared emitting markers that are placed on the moving bodies and connected to a central control unit. Each marker emits a sequential infrared signal and the infrared pulses are captured by camera modules each containing three cameras. The accuracy of the three dimensional measurements is $\pm 0.15 \mathrm{~mm}$ [8]. The system has no hardware-based noise reduction. Wiring harnesses were built for both the rider and the bicycles to facilitate easy bicycle and rider exchange Fig. 2.

The marker coordinates were measured with respect to an inertial frame, $\mathbf{M}$, where the plane normal to $\hat{\mathbf{m}}_{3}$ is coplanar with the treadmill surface and $\hat{\mathbf{m}}_{3}$ is directed upward. We collected the three dimensional locations of 31 markers, 11 of which were located on the bicycle and 20 mapped the rider Fig. 3.

The markers were placed on the bicycle so that we could easily extract the rigid body motion (i.e., body orientations and locations) of the bicycle frame and fork. Four markers were attached to the fork and seven markers were attached to the rear frame. A marker was attached on the right and left sides of the center of each wheel, the seat stays, the ends of the handlebars, and the head tube. A single marker was also attached to the back of the seat post.

We recorded the locations of 20 points on the rider Fig. 3: left and right sides of the helmet near the temple, back of the helmet, shoulders (greater tuberosity of the humerus), elbows (lateral epicondyle of the humerus), wrists (pisiform of the carpus), between the shoulder blades on the spine (T6 of the thoracic vertebrae), the tail bone (coccyx), midpoint on the spine between the coccyx and shoulder blades (L1 on the lumbar vertebrae), hips (greater trochanter of the femur), knees (lateral epicondyle of the femur), ankles (lateral malleolus of the fibula) and feet (proximal metatarsal joint). The body markers were not necessarily placed such that a complete rigid body model could easily be fit to the data. This was done to save setup and processing time because we only wanted a stick figure representation of the rider that allowed us to visually observe the dominant motions of the rider. 


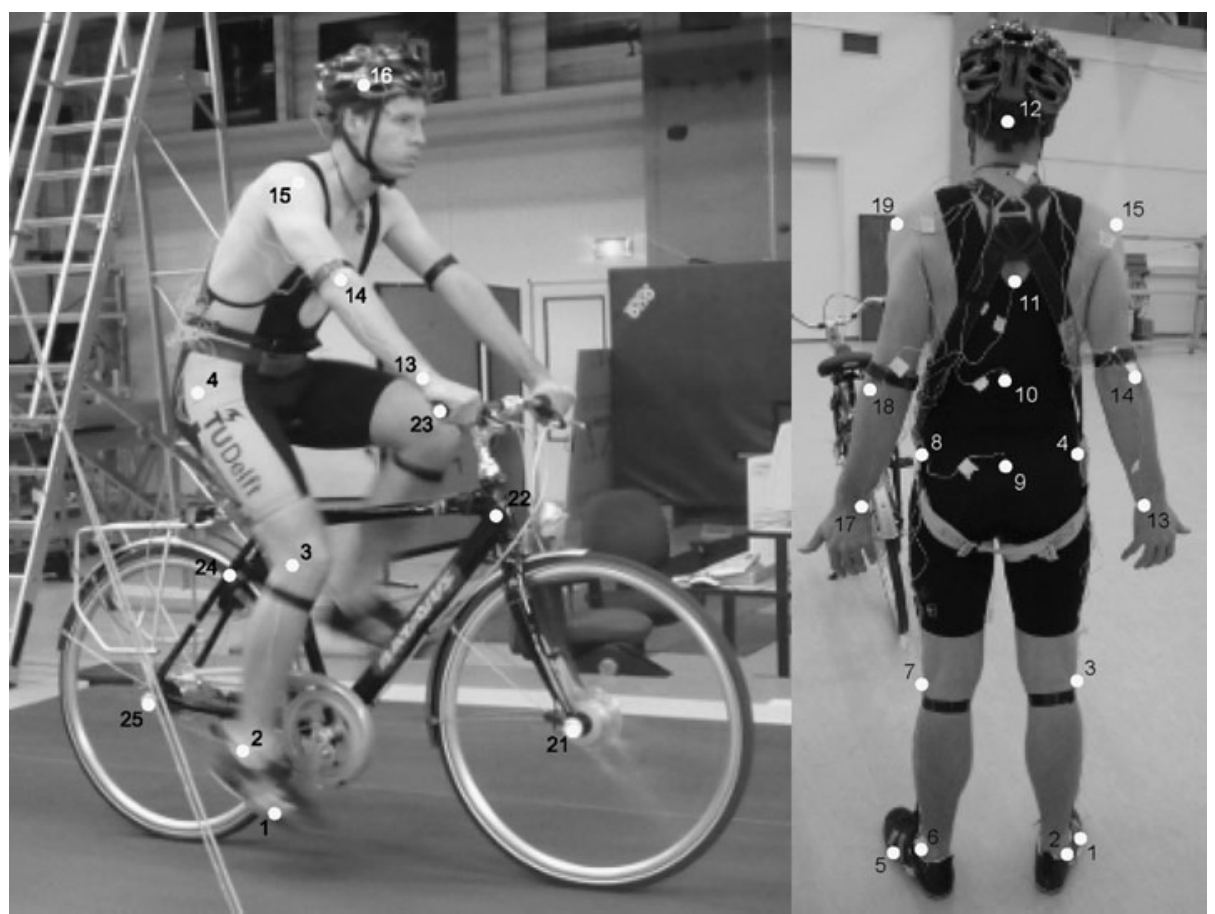

(a)

(b)

Fig. 2 (a) Rider 1 and the Batavus Stratos Deluxe with marker positions. (b) Body marker positions visible from the rear

The stability tasks were designed such that the rider would ride at a constant speed within the range of 2 to $30 \mathrm{~km} / \mathrm{h}$. The bicyclists were told to maintain an upright straight-ahead course on the treadmill and to look into the distance, with exception of the line tracking task. The bicyclists were instructed to bicycle comfortably at the designated speed and data recording was started at random. In all cases, the subject rode at the set speed until comfortable, then data was taken for 60 seconds at a 100 hertz sampling rate. Each task was performed on both bicycles with each rider. The following list describes the various tasks:

Normal pedaling The subject was instructed to simply stabilize the bicycle while pedaling and keep the heading in approximately the forward direction. The speed started at $5 \mathrm{~km} / \mathrm{h}$ and increased in $5 \mathrm{~km} / \mathrm{h}$ increments up to $30 \mathrm{~km} / \mathrm{h}$. The speeds were then decreased in the same fashion to $5 \mathrm{~km} / \mathrm{h}$. From then on the speed was decreased in $1 \mathrm{~km} / \mathrm{h}$ increments until the subject was not able to stabilize the bicycle any longer. Therefore, there were two sets of data for each speed and each bicycle except speeds below $5 \mathrm{~km} / \mathrm{h}$. Several additional runs were also performed with the rider pedaling using a different gear, and thus a different cadence.

Without pedaling This was the same as the normal pedaling task except that a string was attached to the head tube of the bicycle such that the bicycle was fixed 
Fig. 3 Schematic of the marker positions. The rider and bicycle are colored light gray and dark gray, respectively

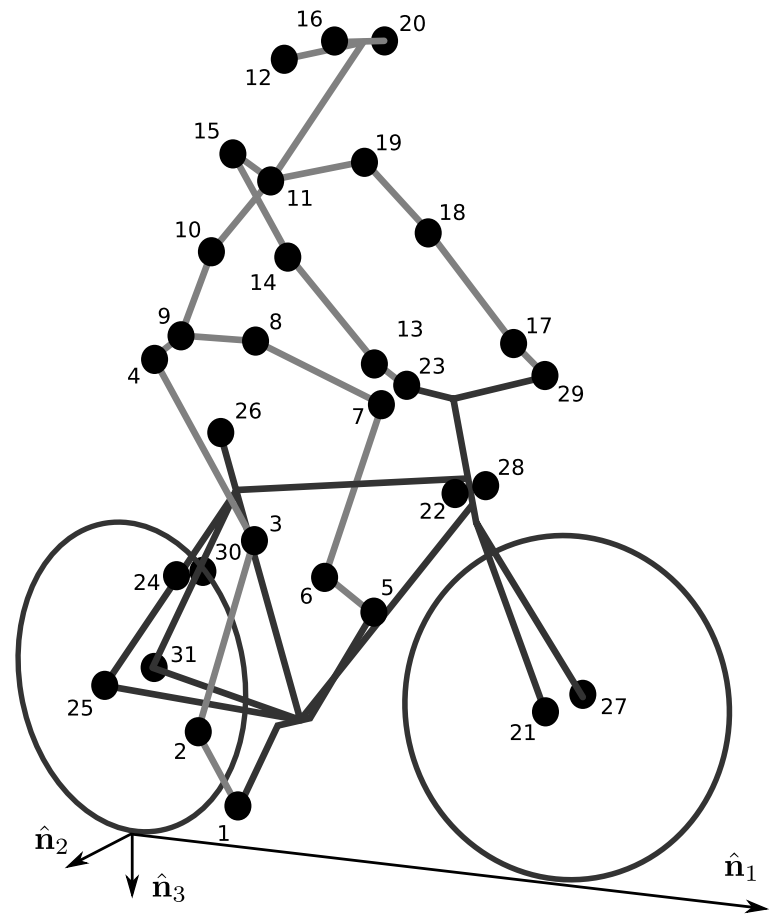

No-hands The riders stabilized the bicycle without using steering for control. They were instructed to keep their hands on their hips while bicycling. The rider started at $30 \mathrm{~km} / \mathrm{h}$ and decreased in $5 \mathrm{~km} / \mathrm{h}$ increments through $20 \mathrm{~km} / \mathrm{h}$ and thereafter the speeds were decreased in 1 or $2 \mathrm{~km} / \mathrm{h}$ increments until the rider was not able to comfortably stabilize the bicycle.

Line tracking This was the same as normal pedaling except that the rider was instructed to track a line on the treadmill surface with the front wheel. A smaller subset of speeds was performed.

These tasks were designed with the intent to answer several questions:

1. What upper body motions are used while bicycling?

2. How does the system motion change with respect to changes in forward speed?

3. How does pedaling influence the control actions?

4. Can the open loop rigid body dynamics be detected in the controlled state?

5. What does the rider do differently to control the bicycle when riding no-hands?

6. Do different bicyclists perform similar motions while performing the same task?

7. Is there a difference in motion when stabilizing and trying to track a line?

Since there is no room to address all of these questions in this paper, we focus on a single rider on the Browser bicycle and two of the tasks: normal pedaling and without pedaling. We were able to draw some conclusions on questions 1 through 4 with this smaller data set. 
Fig. 4 Eigenvalues of the Browser bicycle with the third rider rigidly attached as a function of speed. Note that the initially unstable weave motion becomes stable above $16 \mathrm{~km} / \mathrm{h}$, the weave speed

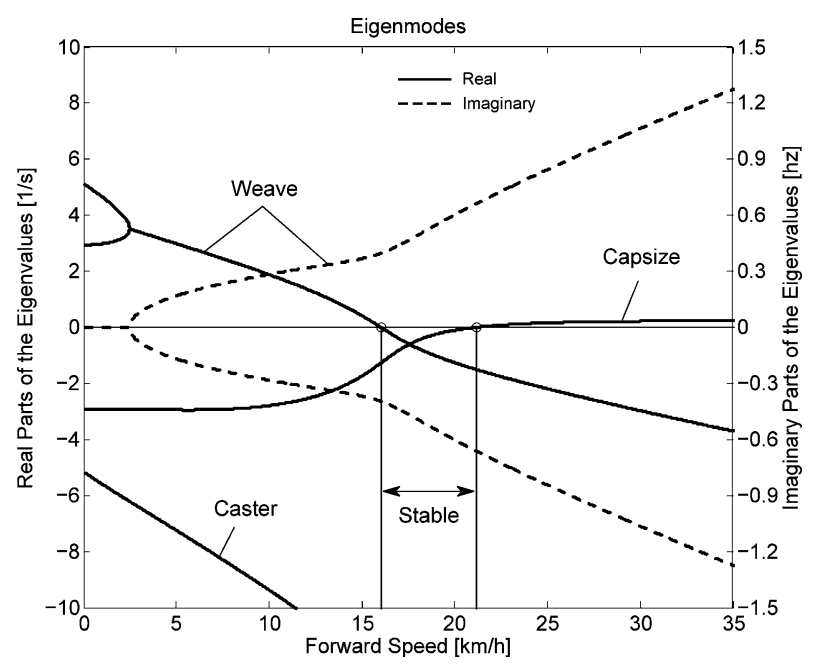

\section{Open loop rigid body dynamics}

One question we have is whether or not the eigenfrequencies of the weave motion for the uncontrolled system can be detected in the results from the stabilization tasks. In order to predict the uncontrolled (open loop) eigenvalues of the rigid rider system, the basic geometry, mass, center of gravity locations, and moments of inertia of the bicycle were measured. Also, the riders were measured and weighed such that the body segment geometry, mass, center of gravity locations, and moments of inertia could be estimated. The physical parameter estimation methods are described in [9]. This data was used to calculate eigenvalues and eigenvectors of the uncontrolled open loop system Fig. 4.

\section{Data processing}

\subsection{Missing markers}

The Optotrak Certus Motion Capture System [8] is based on the cameras' ability to detect the infrared light from the sensors so there are occasional gaps in the coordinate data due to the markers going out of view. We attempted to minimize this by careful marker and camera placement but were not able to totally eliminate the error. Any missing markers on the bicycle were reconstructed using the assumption that the bicycle is a rigid body. We had more than three markers on both the frame and fork, so if one marker location was not detected we used the relative location of the remaining markers to reconstruct the missing marker. The gaps in the data of the markers on the human were repaired by fitting a cubic spline through the data. The spline estimated the marker coordinates during the gaps. We only used the splined data if the gaps were less than 10 time steps, or $0.1 \mathrm{sec}$; otherwise the trials were discarded.

\subsection{Relative motion}

We were interested in the analysis of three different marker combinations: the bicycle alone, the rider relative to the bicycle and the bicycle and rider together. The motion of the bicycle 


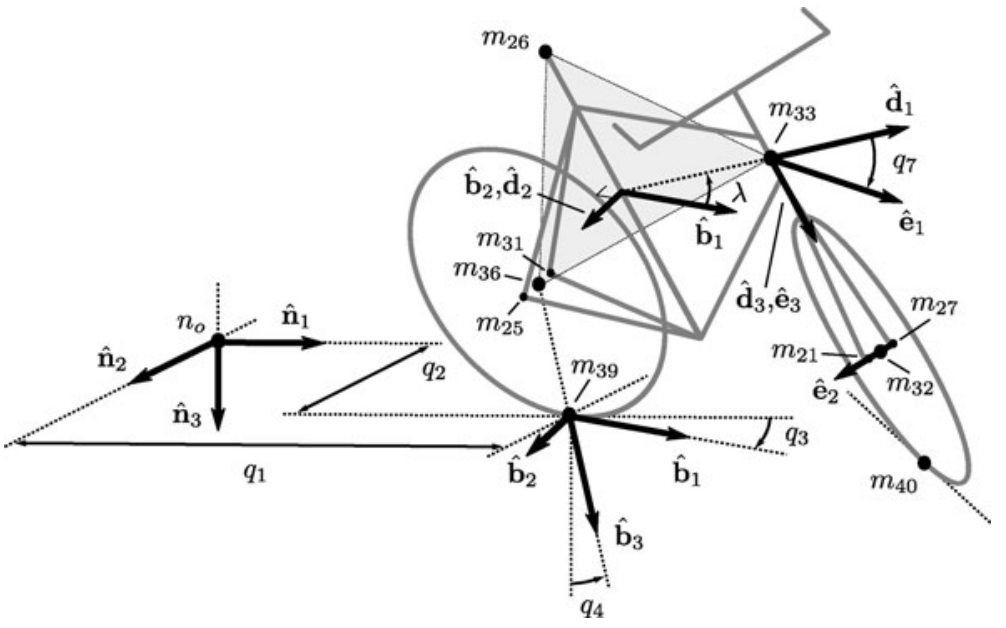

Fig. 5 Diagram of the bicycle's inertial frame $\mathbf{N}$, rear frame $\mathbf{B}$, front frame $\mathbf{E}$ and configuration variables

and the bicycle-rider were calculated with reference to the $\mathbf{N}$ inertial frame ${ }^{1}$ and the motion of the rider was calculated with respect to the rear frame of the bicycle B Fig. 5. These three marker combinations allowed us to differentiate more easily between rider specific and bicycle specific motions. Furthermore, six of the variables that describe the configuration of the bicycle in time were calculated to give insight into the rigid body dynamics. The configuration variables $q_{1}$ and $q_{2}$ locate the contact point of the rear wheel of the bicycle. The $\mathbf{B}$ frame captures the yaw $\left(q_{3}\right)$ and roll $\left(q_{4}\right)$ motions of the bicycle frame, the $\mathbf{D}$ frame is an intermediate frame that differs from $\mathbf{B}$ only by the bike's headtube angle $(\lambda)$, and the $\mathbf{E}$ frame captures the steering angle $\left(q_{7}\right)$ of the bicycle fork relative to the bicycle frame. The pitch of the bicycle frame $\left(q_{6}\right)$ is assumed to be zero. Details of these calculations are shown in Appendix.

\subsection{Principal component analysis}

We used Principal Component Analysis, PCA, [10] to extract and characterize the dominant motions of the system. Calculating the principal components effectively transforms the space of the data to a space that maximizes the variance of the data. The typical advantage of PCA is that the dimension of the system can be reduced and still retain enough information to adequately describe the system. We are primarily interested in the way that PCA is able to extract linear components and rank them in order of variance from the mean position. If we assume that the components with the largest kinematic variance are motions that are the dominant motions used for control and propulsion (which in general is not necessarily true for dynamical systems) the comparison of these components for different riding conditions can give insight into what motions may be important for developing a biomechanical control model of the bicyclist.

\footnotetext{
${ }^{1}$ The $\mathbf{N}$ frame is used instead of the $\mathbf{M}$ frame to comply with the vehicle coordinate standards used in [1]. See Appendix for the derivation.
} 
The repaired data from the motion capture measurements contained the $x, y$, and $z$ coordinates of each marker 1 through $l$ at each time step $j=1,2, \ldots, n$. Each marker has three coordinates so there are a total of $m=3 l$ coordinates $i=1,2, \ldots, m$. The coordinates at each time step can be collected in vector $\mathbf{p}_{j}$.

$$
\mathbf{p}_{j}^{T}=\left[\begin{array}{lllllllll}
x_{1 j} & \cdots & x_{l j} & y_{1 j} & \cdots & y_{l j} & z_{1 j} & \cdots & z_{l j}
\end{array}\right]=\left[\begin{array}{llll}
p_{1 j} & p_{2 j} & \cdots & p_{m j}
\end{array}\right]
$$

We can organize these coordinate vectors into a matrix, $\mathbf{P}$, where the rows, $i$, map a single coordinate of a marker through $n$ time steps.

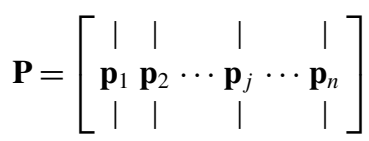

The principal components were calculated for the three marker combinations as described earlier where $n=60 \times 100=6000$ time steps. The number of rows of $\mathbf{P}$ were $(m=3 \times 31=93),(m=3 \times 11=33)$ and $(m=3 \times 20=60)$ for the bicycle-rider, the bicycle alone and the rider alone, respectively.

One method of determining the principal components is to calculate the eigenvectors of the covariance matrix of the mean-subtracted data. We begin by calculating the mean $\mathbf{u}$ (1) of the rows of $\mathbf{P}$ and subtracting it from each column of $\mathbf{P}$ to form the mean-subtracted data matrix $\overline{\mathbf{P}},(2)$.

$$
\mathbf{u}=\frac{1}{n} \sum_{j=1}^{n} \mathbf{p}_{j}
$$

A vector of ones

$$
\mathbf{h}^{T}=\left[\begin{array}{llllll}
h_{1} & h_{2} & \cdots & h_{j} & \cdots & h_{n}
\end{array}\right] \quad \text { where } h_{j}=1 \text { for all } j
$$

allows us to subtract $\mathbf{u}$ from each column of $\mathbf{P}$,

$$
\overline{\mathbf{P}}=\mathbf{P}-\mathbf{u h}^{T}
$$

The covariance matrix $\mathbf{C}$ of $\overline{\mathbf{P}}$ can then be calculated with (3).

$$
\mathbf{C}=\frac{1}{n-1} \overline{\mathbf{P}} \overline{\mathbf{P}}^{T}
$$

Calculating the eigenvectors $\mathbf{v}_{i}$ and eigenvalues $\lambda_{i}$ of the covariance matrix effectively transforms the space to one where the variances are maximized and the covariances are zero. The eigenvectors are the principal components of the data set and the corresponding eigenvalues represent the variance of each principal component. The eigenvectors are ordered by decreasing eigenvalue where $\mathbf{v}_{1}$ is the eigenvector corresponding to the largest eigenvalue. The eigenvalues and eigenvectors are calculated by finding the independent solutions to (4).

$$
\mathbf{C v}_{i}=\lambda_{i} \mathbf{v}_{i}
$$

Each time step can now be represented as a linear combination of the principal components.

$$
\mathbf{p}_{j}=\mathbf{u}+a_{1 j} \mathbf{v}_{1}+a_{2 j} \mathbf{v}_{2}+\cdots+a_{m j} \mathbf{v}_{m}
$$


The coefficients $a_{i j}$ can be solved at each time step $j$ by reformulating (5) and solving the system of linear equations.

$$
\mathbf{P}-\mathbf{u h}^{T}=\left[\begin{array}{cccc}
\mid & \mid & & \mid \\
\mathbf{v}_{1} & \mathbf{v}_{2} & \ldots & \mathbf{v}_{m} \\
\mid & \mid & & \mid
\end{array}\right]\left[\begin{array}{ccc}
a_{11} & \ldots & a_{1 n} \\
\vdots & \ddots & \vdots \\
a_{m 1} & \ldots & a_{m n}
\end{array}\right]=\mathbf{V A}
$$

and

$$
\mathbf{A}=\mathbf{V}^{-1}\left(\mathbf{P}-\mathbf{u h}^{T}\right)
$$

With the principal components $\mathbf{v}_{i}$ being constant, the behavior in time is described by the coefficients $a_{i j}$ where the discretization in time is indexed by $j$. The order of the system can be reduced by eliminating principal components that have little variance. We arbitrarily decided to examine the first $k=10$ principal components knowing that the first five would be based around the larger motions such as pedaling and that the remaining five may reveal some of the motions associated with control. The variance of each component, $\operatorname{var}\left(\mathbf{a}_{i}\right)=\lambda_{i}$, is summed to determine the cumulative percentage of variance of the principal components, $g_{k}$.

$$
g_{k}=100 \frac{\sum_{i=1}^{k} \lambda_{i}}{\sum_{i=1}^{m} \lambda_{i}} \quad \text { where } 1 \leq k \leq m
$$

Highly correlated data will show that even when $k \ll m, g_{k}$ is close to $100 \%$. Using 10 components $g_{10}$ covers $100 \%$ (standard deviation, $\sigma=10^{-14} \%$ ) of the variation in the data for the bicycle, rider and bicycle-rider. The matrix $\mathbf{A}$ can then be reduced to a $k \times n$ matrix and eigenvectors greater than $\mathbf{v}_{k}$ can be eliminated.

\subsection{Data visualization}

We developed a Graphical User Interface, GUI, in MATLAB that easily allows different trials to be compared with one another Fig. 6. The program loads in two different trials along with information on each trial. A graphical representation of the rider and bicycle are displayed in two adjacent screens and can be viewed from multiple perspectives. The animations of the runs can be played at different speeds, rewound and fast forwarded. The principal components are shown beside the corresponding animation display and combinations can be turned on and off for identification and comparison. Frequency and amplitude information for the temporal coefficients $a_{i j}$ can also be displayed for comparison.

\section{Results}

\subsection{Motion identification}

The reduced set of data provides two important pieces of information for the identification of motion: the principal components $\mathbf{v}_{i}$ and the corresponding coefficients $a_{i j}$. The principal components represent linear trajectories of the markers and the coefficients show how the markers follow the trajectories with time. We began processing the data by reviewing each principal component of each trial in the GUI and noting what type of motion we saw Table 1 . These descriptions were subjective because we grouped marker movement based on 


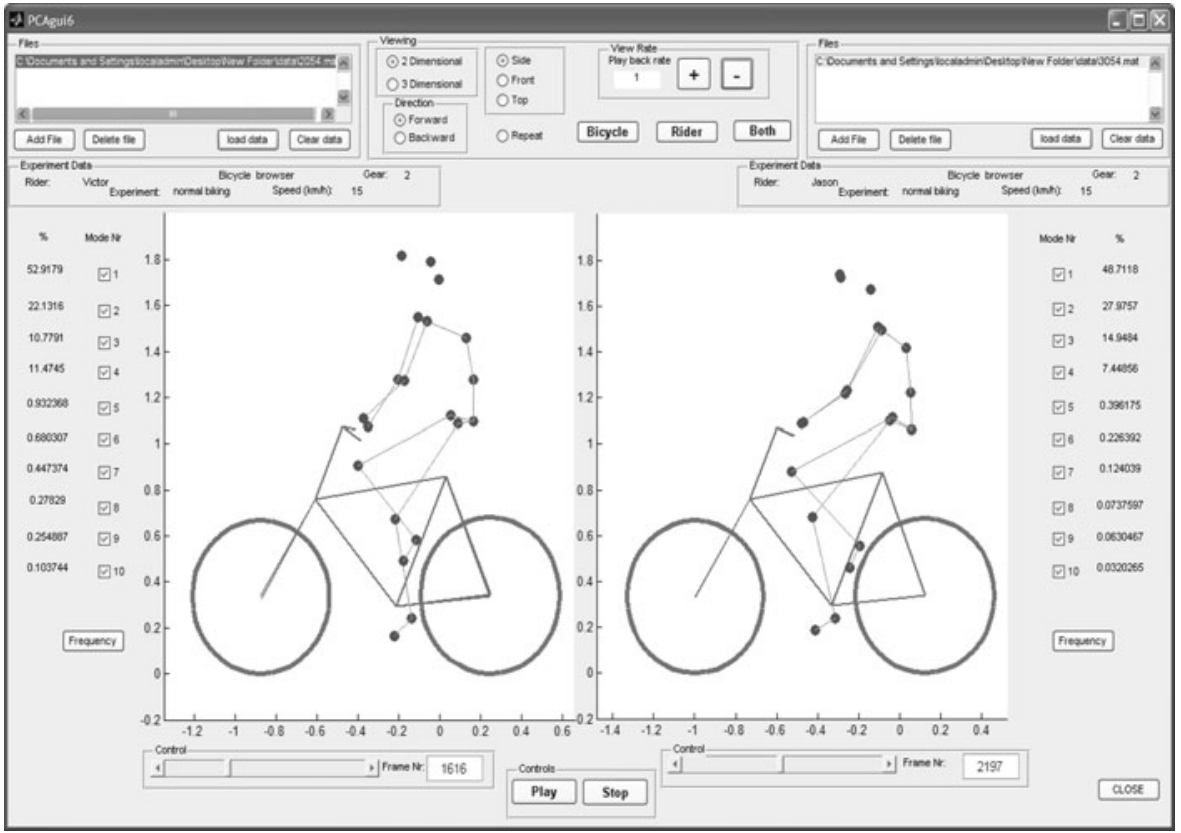

Fig. 6 Screen shot of the MATLAB graphical user interface (GUI) used to visualize principal components and compare between different components and trials

Table 1 Example raw trial description for the bicycle and rider during normal pedaling at $10 \mathrm{~km} / \mathrm{h}$

$i \quad \%$ Variance Motion description Frequency description

\begin{tabular}{|c|c|c|c|}
\hline 1 & 45.50 & primarily longitudinal motion, some lateral & $\begin{array}{l}\max \text { amp }=0.6 \mathrm{~m}, \text { most freq below } 0.5 \mathrm{~Hz} \text {, } \\
\text { tiny spike at } 1.6 \mathrm{~Hz}\end{array}$ \\
\hline 2 & 29.39 & $\begin{array}{l}\text { primarily lateral motion, some longitudi- } \\
\text { nal, small feet motion }\end{array}$ & $\begin{array}{l}\max \text { amp }=0.35 \mathrm{~m} \text {, little spike at } 0.8 \mathrm{~Hz} \\
\text { most freq below } 0.5 \mathrm{~Hz}\end{array}$ \\
\hline 3 & 15.41 & $\begin{array}{l}\text { vertical pedaling, slight spine bend, hip/ } \\
\text { head/shoulder sway out of phase with ped- } \\
\text { aling }\end{array}$ & $\begin{array}{l}\max \operatorname{amp}=0.27 \mathrm{~m} \text {, large dominant spike } \\
\text { at } 0.8 \mathrm{~Hz}\end{array}$ \\
\hline 4 & 8.27 & horizontal pedaling, head/shoulder sway & $\begin{array}{l}\text { large dominant spike at } 0.8 \mathrm{~Hz} \text { with } 0.19 \mathrm{~m} \\
\text { amp }\end{array}$ \\
\hline 5 & 0.82 & yaw, knees stay still & $\begin{array}{l}\max \operatorname{amp}=0.04 \mathrm{~m} \text { at } 0.33 \mathrm{~Hz}, \text { most freq } \\
\text { below } 1 \mathrm{~Hz}\end{array}$ \\
\hline 6 & 0.27 & erratic left-hand movement & $\max \mathrm{amp}=0.018 \mathrm{~m}$, most freq below $2 \mathrm{~Hz}$ \\
\hline 7 & 0.21 & steer, left-hand movement, slight roll & $\begin{array}{l}\text { most freq below } 2 \mathrm{~Hz} \text {, spike at } 0.33 \mathrm{~Hz} \text { and } \\
1.58 \mathrm{~Hz}\end{array}$ \\
\hline 8 & 0.07 & knee and head bounce & dominant spike at $1.58 \mathrm{~Hz}$ \\
\hline 9 & 0.04 & lateral knee movement, head jiggle & $\begin{array}{l}\text { spikes at } 1.58 \mathrm{~Hz} \text { and } 2.37 \mathrm{~Hz} \text {, most freq } \\
\text { below } 2.5 \mathrm{~Hz}\end{array}$ \\
\hline 10 & 0.02 & head and knee jiggle & $\begin{array}{l}\text { spikes at } 1.58 \mathrm{~Hz} \text { and } 3.17 \mathrm{~Hz} \text {, most freq } \\
\text { below } 3.5 \mathrm{~Hz}\end{array}$ \\
\hline
\end{tabular}


Fig. 7 Coefficients $a_{i j}$ versus time of the first five principal components for normal pedaling at $10 \mathrm{~km} / \mathrm{h}$

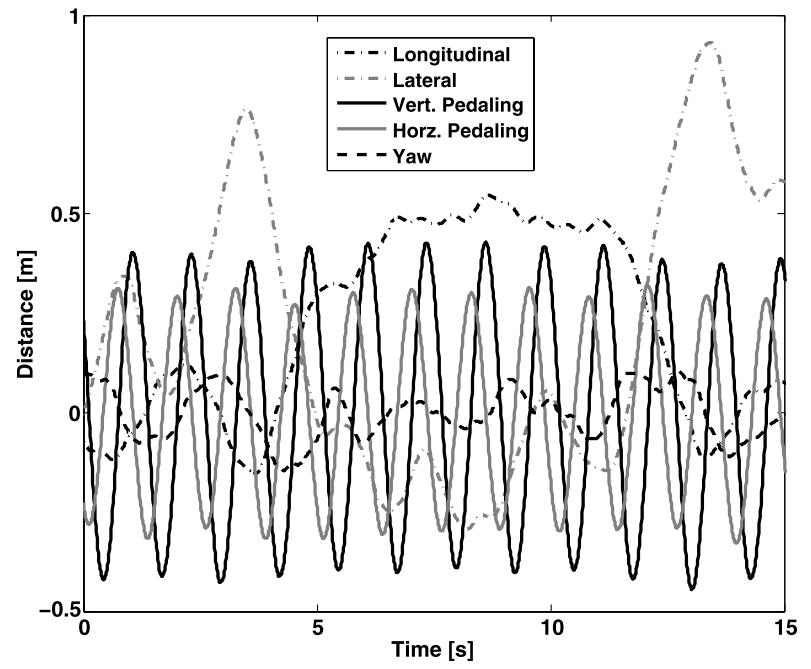

Fig. 8 The frequency content of the first five principal components for normal pedaling at $10 \mathrm{~km} / \mathrm{h}$. The vertical black line represents the open loop weave frequency $(0.28 \mathrm{~Hz})$ determined from Fig. 4 at this forward speed. The pedaling frequency is about $0.8 \mathrm{~Hz}$ at this speed; see Fig. 11

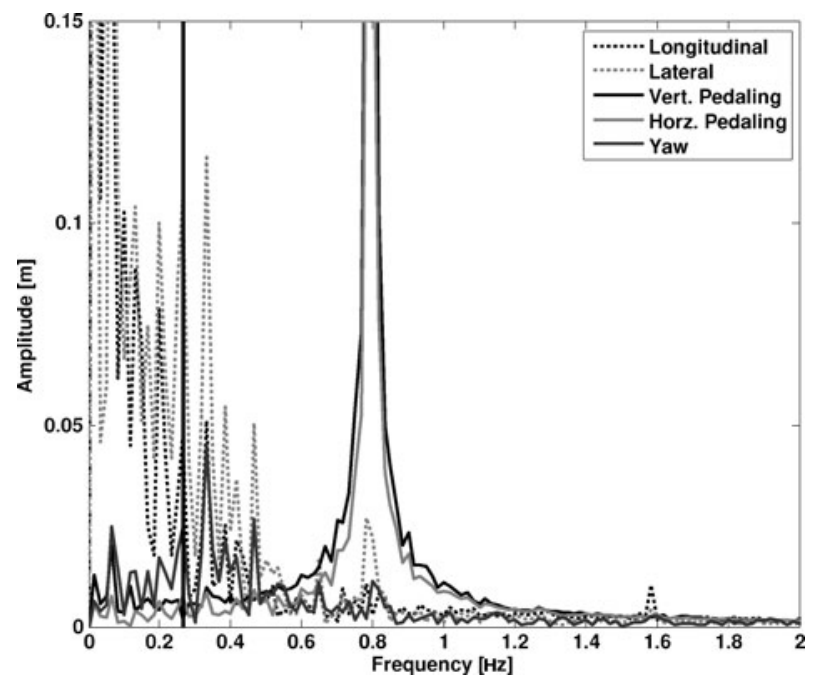

our preconceived understanding of rider and bicycle motion. Some of the components displayed motions that were not physically possible such as the upper leg stretching in length during the knee bounce. This is possible when examining a single component but when superimposed over the rest of the components the unrealistic motions are not present. Furthermore, for each component we examined amplitude and frequency content of the associated coefficients $a_{i j}$ as shown in Figs. 7 and 8 and noted the shape of the frequency spectrum and the frequencies at any distinct spikes.

Several conclusions can be drawn from examining the coefficient data. First, some of the components are linked by the frequencies of the coefficients and describe an identifiable motion. The most obvious of these is that the vertical and horizontal pedaling components make up the circular pedaling motion. Both vary periodically and have a dominant frequency which is defined by the cadence. In the example trial, Table 1, the upper body motions are also linked to the pedaling. Components 8 and 9 both correspond to a frequency that is 
twice the pedaling frequency, which may be due to the forces created during each pedal stroke. Component 6 seems to be the result of a bad marker signal. Components 5 and 7 are interesting because they display motions of the bicycle that are not dominated by the pedaling frequency and may be candidate control motions. The percentage variance of each component gives an idea of the relative amplitude of the components. The descriptions of each trial were used to compile a list of motions that contribute to the principal components. These motions, illustrated in Fig. 9, are:

Drift The bicycle and rider drift longitudinally and laterally on the surface of the treadmill. The motions are typically defined by two components that are not necessarily orthogonal or aligned with the inertial coordinate system. The motion is random and at low frequencies.

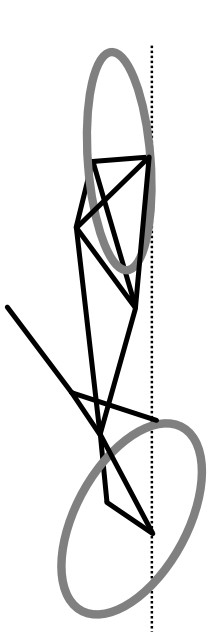

(a)

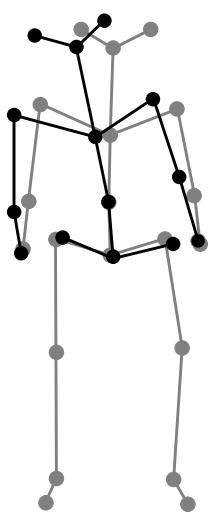

(e)

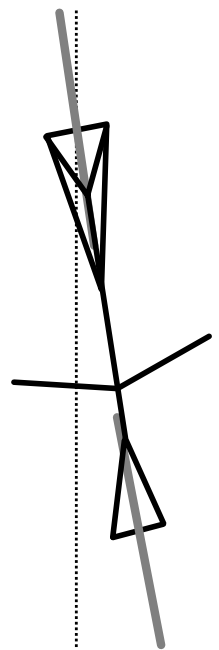

(b)

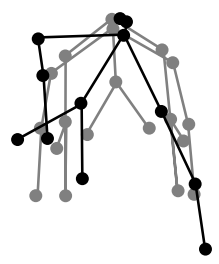

(f)

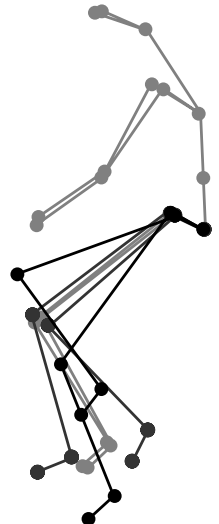

(c)

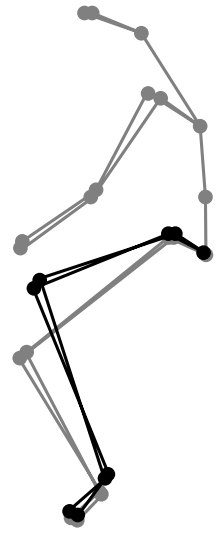

(g)

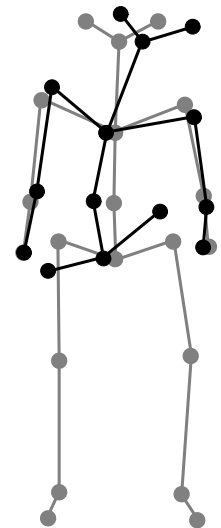

(d)

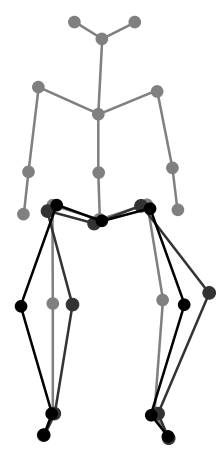

(h)

Fig. 9 Diagrams of the common motions. (a) Top view of bicycle steer and roll, (b) bicycle yaw, (c) horizontal and vertical components of pedaling, (d) spine bend, (e) rider lean, (f) top view of rider twist, (g) knee bounce and (h) two lateral knee motions. All but pedaling (c) are exaggerated for clarity 
Steer Rotation of the front assembly with respect to the rear frame. The steering may appear linked to one of the pedaling components at the pedaling frequency or may be in one or more components sometimes combined with roll and/or yaw at more random frequencies, Fig. 9(a).

Roll The bicycle and the rider roll with respect to the ground plane. Roll is typically linked with steer and/or yaw and often at the pedaling frequency, Fig. 9(a).

Yaw The heading angle of the bicycle and rider change together with respect to the ground plane. This is typically linked with steer, roll, and/or the drift, Fig. 9(b).

Pedaling This motion is defined by two or more components, typically a vertical and horizontal motion of the feet, that show the feet rotating around the crank axle at a distinct frequency and the legs following suit, Fig. 9(c).

Bend The spine bent laterally and was always connected with the vertical pedaling component, Fig. 9(d).

Lean The upper body, shoulders and head lean laterally with respect to the rear frame and was always linked with the horizontal pedaling component, Fig. 9(e).

Twist The shoulders rotate about the torso axis. This was linked to components that contained steering motions, both random and at the pedaling frequency, Fig. 9(f).

Bounce The knee markers bounce up and down, the back straightens and the head nods at twice the pedaling frequency, Fig. $9(\mathrm{~g})$.

Knees The knees move laterally relative to the bicycle frame in both opposing directions and the same direction at random low frequencies, Fig. 9(h).

Head Head twists and random head motions showed up often. These seemed to be due to the rider looking around randomly.

\subsection{Motion characterization}

To identify how bicycling changes with speed it would be ideal to investigate how the amplitude of each component varies with speed. However, the analysis does not return the same set of components for each run so such a comparison is typically not possible. Therefore, components were grouped into classes, where each class shows a specific physically relevant motion. The same total motion of the class can be described by one set of components in one trial and another, probably different, set of components in another trial. How the amplitudes of these classes vary among experiments can be used as a measure for how the rider and bicycle motion varies among trials.

To objectively identify which coefficients show the same type of motion and could therefore form a class, the frequency content of each of the time coefficients in a single trial was correlated to that of each of the other components in that trial. Next, a minimum correlation value was set to determine which coefficients were correlated to each other. When the minimum was set at 0.9 only the coefficients making up the pedaling motion could be considered correlated. On the other hand, when a minimum level of 0.7 was used practically every coefficient was correlated to each other. The only exception was the coefficient that displayed the bounce. Its maximum correlation with another coefficient was no higher than 0.4 for any of the tested speeds. The 0.8 level gave a number of distinct classes of components, and thus this level was used to identify which coefficients were connected. Finally, the correlated coefficients were viewed simultaneously in the GUI enabling the determination of the motion class.

The correlated coefficients were used to form six different classes of motions, Table 2, each made up of combinations of the previously described motions in Fig. 9. 
Table 2 The six primary motion classes

Fig. 10 The relative percent variance of the four classes: Pedaling, Steer-Yaw-Roll, Bounce and Knees, at the different speeds when the Drift and Other classes were removed from the results for normal pedaling. The solid lines are scaled to $100 \%$ (left axis), the dotted lines are scaled to $10 \%$ (right axis)

\begin{tabular}{ll}
\hline Class name & Class description \\
\hline Drift & Drift \\
Pedaling & Pedaling 9(c), Bend 9(d), Lean 9(e), Twist 9(f) \\
Steer-Yaw-Roll & Steer and Roll 9(a), Yaw 9(b) \\
Bounce & Bounce 9(g) \\
Knees & Knees 9(h) \\
Other & Head and components that showed noise of \\
& some sort \\
\hline
\end{tabular}

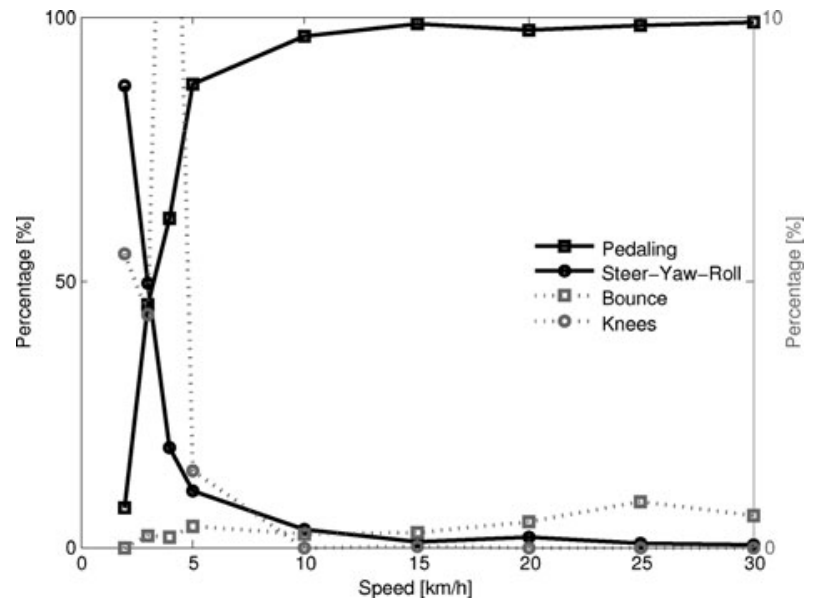

In most cases, the correlated coefficients described a single class. However, sometimes, this was not the case and the coefficients were used to describe more than one class. An example is that at low speed the components containing the drift motions also contained large steer, yaw, and roll motions. Therefore, the motions were placed in both the Drift and the Steer-Yaw-Roll classes.

Since the rider was not instructed to hold a specific location on the treadmill the Drift class, which was usually the class with the largest amplitude, was not used in further analysis of the motion and neither was the 'Other' class. For each of the remaining classes, the percentages of variance of the remaining components were recalculated without the components placed in the Drift and the Other classes.

We also calculated various configuration variables from the bicycle marker locations (see Appendix) independent of the PCA perspective for more specific motion characterizations. This allowed us to investigate the bicycle's configuration variable time histories and frequency content explicitly.

\subsection{Characterization of motions during normal pedaling}

Figure 10 shows how the relative percent variance of the four classes: Pedaling, SteerYaw-Roll, Bounce and Knees varies with speed for Rider 3 on the Batavus Browser bicycle. The percentage is the average of two runs at speeds $5 \mathrm{~km} / \mathrm{h}$ and above. From the 


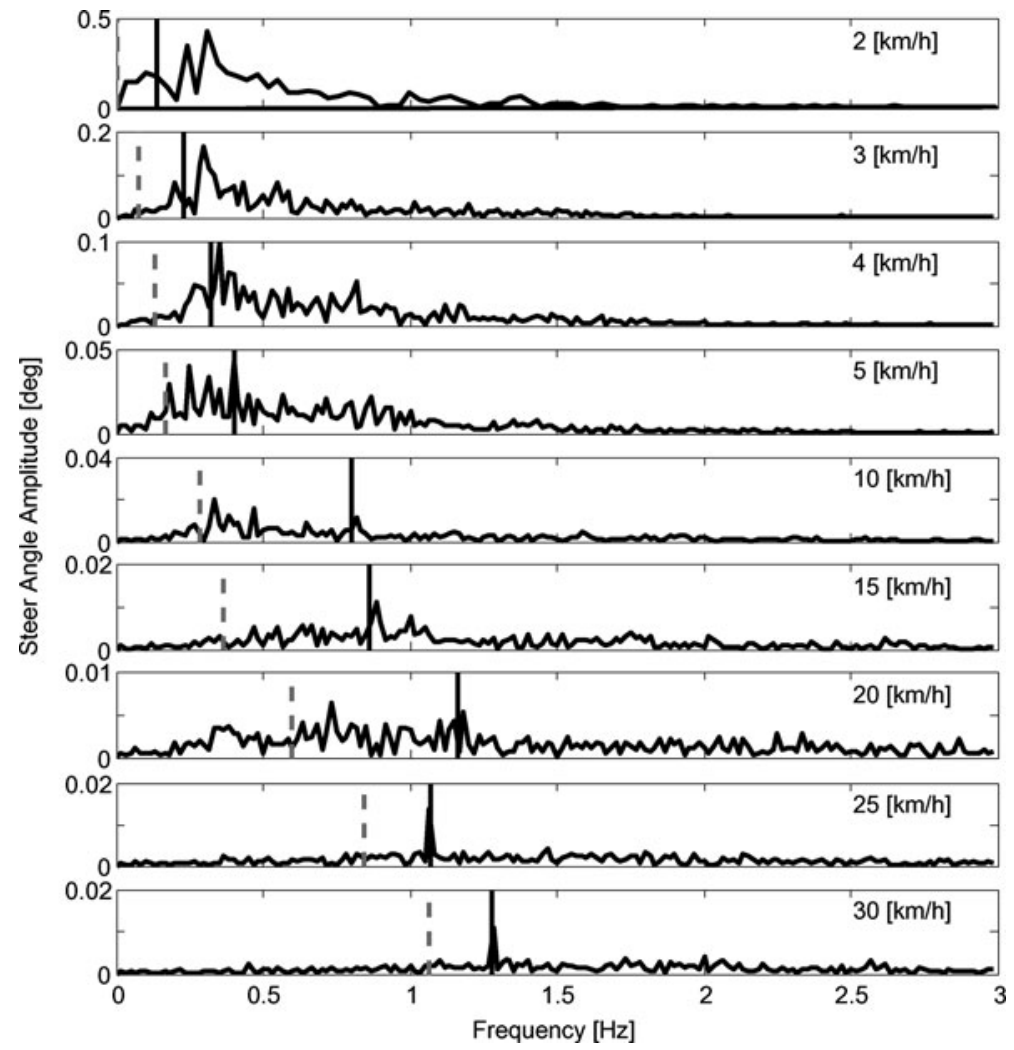

Fig. 11 Steer angle amplitude plot for the nine different speeds for normal pedaling experiment. Solid vertical line indicates the pedaling frequency. Dashed vertical gray line indicates the bicycle-rigid rider open loop weave eigenfrequency from Fig. 4

graph, it is clear that at $10 \mathrm{~km} / \mathrm{h}$ and higher speeds practically all the motion that is taking place is the pedaling motion class. Below $10 \mathrm{~km} / \mathrm{h}$, the Steer-Yaw-Roll class becomes increasingly active and the relative percentage of the motion taking place in the pedaling class drops. Also, at speeds below $10 \mathrm{~km} / \mathrm{h}$, the lateral knee motion (Knees) class percentage increases with decreasing speed. The increase is not as significant as that of the Steer-Yaw-Roll class (increase to roughly $5 \%$ at $2 \mathrm{~km} / \mathrm{h}$ ), but it is certainly visible. The spike at $4 \mathrm{~km} / \mathrm{h}$ can be attributed to the fact that the classes may contain higher variance motions because the classification method is based on principal components that are not necessarily consistent between runs. The Bounce roughly remains constant at all speeds.

The steer angle amplitude-frequency plot for each of the speeds calculated from the bicycle rigid body motions is given in Fig. 11. It clearly shows that the steering actions take place at or around the pedaling frequency for high and low speeds, respectively. It also shows that the amplitude of the steering angle increases by $5000 \%$ when the speed decreases from $30 \mathrm{~km} / \mathrm{h}$ to $2 \mathrm{~km} / \mathrm{h}$. Figure 11 also shows the open loop, rigid rider, weave eigenfrequency for each speed obtained from Fig. 4. Apparently the open loop eigenfrequency is not a frequency at which the bicycle-rider operates. 
Fig. 12 The percent variance of each of the three classes: Steer-Yaw-Roll, Bounce and Knees, at the different speeds when the Drift and Other classes were removed from the results for trials without pedaling. The solid lines are scaled to $100 \%$ (left axis), the dotted lines are scaled to $15 \%$ (right axis)

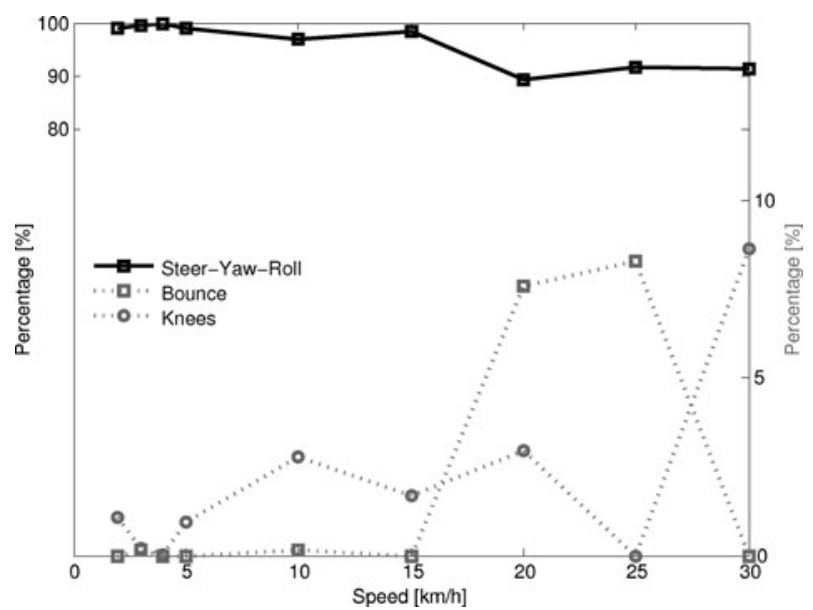

\subsection{Characterization of motions without pedaling}

During normal pedaling, all motions, including the control tasks, are dominated by the pedaling motions. Therefore we also looked at the motions of bicycle-rider system without the influence of pedaling. Figure 12 shows how the percent variance of Steer-Yaw-Roll, Bounce and Knees varies with speed for Rider 3 on the Batavus Browser bicycle without pedaling. Since the bicycle is towed and the riders feet remain in the same, constant, position relative to bicycle, there is no pedaling class present in analysis. Furthermore, no bend, lean or twist motions with high variance were detected during the experiments. It is clear that at all speeds most motion takes place in the Steer-Yaw-Roll class. Also interesting is that, unlike in the normal pedaling situation, the Knee motion percentage does not increase at low speeds. This may mean that the lateral knee motion is connected to pedaling in some way. Like for the pedaling case, the Bounce and Knee classes may contain different principal components and a statistical approach to evaluate the percent variance of the classes would provide clearer results. Also note that as the bicycle becomes self stable above $16 \mathrm{~km} / \mathrm{h}$ the total variance is tiny and thus any sort of random knee motion can be a relatively large motion.

Figure 13 shows the bicycle rigid body steer angle frequency-amplitude plot for different speeds. Compared to normal pedaling, the amplitudes are about half the size at the low speeds and one tenth the size at high speeds, indicating that smaller steering angles were made. The frequency content now also shows a much wider, flatter spectrum compared to normal pedaling. At 10 and $15 \mathrm{~km} / \mathrm{h}$, the frequency with the largest amplitude is near the open loop weave eigenfrequency. However, at the other speeds, this is not the case, once again indicating that the rigid body open loop weave eigenfrequency is not the frequency at which the bicycle is controlled.

\section{Conclusions}

The view provided by principal component analysis into bicycle-rider interaction, biomechanics and control has led us to several conclusions. During normal bicycling there are 


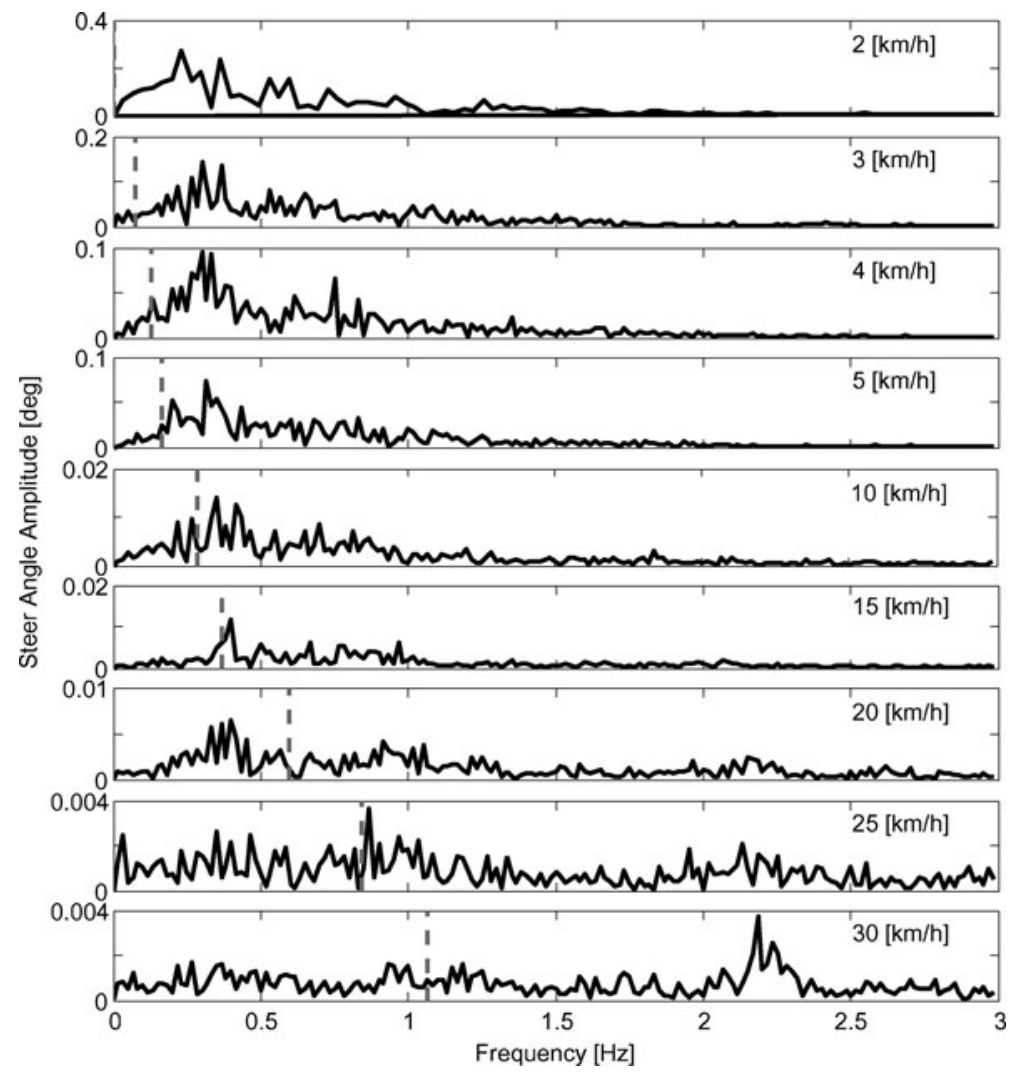

Fig. 13 Steer angle amplitude plot for the nine different speeds for the tasks without pedaling. Dashed vertical grey line indicates the bicycle-rigid rider open loop weave eigenfrequency obtained from Fig. 4

several dominant upper body motions: lean, bend, twist and bounce, all of which seem to be linked to the pedaling motion. This is important for understanding which inputs are related to fundamental balance control and which are reactions to pedaling. We hypothesize that lateral control is mainly accomplished by steering since only upper body motion was observed at the pedaling frequency. If upper body motions are used for control then this control is carried out at the pedaling frequency. Considering variations of motion with respect to speed, we observed that there is a great deal of steering at low speeds but this decreases in magnitude as speed increases. This is generally true for all motions and shows that the bicycle-rider system becomes more stable at higher speeds with few detectable control actions. At low speeds additional lateral knee motions are observed which are probably more effective at augmenting steering control for lateral balance than upper body motions.

The bicycle model predicts that the weave mode is stable above about $16 \mathrm{~km} / \mathrm{h}$. Intuition might possibly lead one to believe, if the weave mode is already stable, that the weave frequency might be relatively undisturbed by rider control actions and therefore present in the closed loop dynamics. However, we found no evidence of a distinct weave frequency in the steer angle time histories of any run. In fact, the only distinct frequency that sometimes appeared was the pedaling frequency. 
Principal component analysis provided a unique view into the control actions of a rider on a bicycle, but limitations in data reduction and motion grouping leave room for more objective statistical views into the motion of the bicycle-rider system.

Acknowledgements We would like to thank Knoek van Soest and Richard Casius of the Faculty of Human Movement Sciences at the Vrije Universiteit, Amsterdam, for their cooperation and use of their equipment for the experiments and for Richard's expertise and help in operating the motion capture system and data processing. Also, we thank Victor Ahlm for a tireless day of bicycling and the Dutch bicycle manufacturer, Batavus, for supplying the bicycles.

Open Access This article is distributed under the terms of the Creative Commons Attribution Noncommercial License which permits any noncommercial use, distribution, and reproduction in any medium, provided the original author(s) and source are credited.

\section{Appendix: Inertial frames and configuration variables}

The transformation from marker coordinates to rigid body inertial frames and configuration variables shown in Fig. 5 is described here. A reference frame, $\mathbf{N}$, with origin $n_{o}$ corresponding with the benchmark bicycle [1] is defined with respect to the Optotrak reference frame, $\mathbf{M},(9)$.

$$
\mathbf{N}=\left[\begin{array}{l}
\hat{\mathbf{n}}_{1} \\
\hat{\mathbf{n}}_{2} \\
\hat{\mathbf{n}}_{3}
\end{array}\right]=\left[\begin{array}{rrr}
1 & 0 & 0 \\
0 & -1 & 0 \\
0 & 0 & -1
\end{array}\right]\left[\begin{array}{c}
\hat{\mathbf{m}}_{1} \\
\hat{\mathbf{m}}_{2} \\
\hat{\mathbf{m}}_{3}
\end{array}\right]
$$

Thirty-one marker locations were recorded and the vector to each is defined as $\mathbf{r}^{m_{k} / n_{o}}$ where $k=1,2, \ldots, l$ for the original markers and $k=l+1, \ldots$ for any additional virtual markers. To calculate the reference frame attached to the rear bicycle we formed a frame center plane from the seat post marker, $m_{26}$, and two new additional virtual markers at the center of the rear wheel, $m_{36}$, and the center of the head tube, $m_{33}$. For example, the center of the rear wheel was calculated by (10) where $m_{25}$ and $m_{31}$ are the left and right rear wheel markers.

$$
\mathbf{r}^{m_{36} / n_{o}}=\left(\mathbf{r}^{m_{25} / n_{o}}+\mathbf{r}^{m_{31} / n_{o}}\right) / 2
$$

The normal vector to the plane through the rear wheel center, seat post, and the head tube center is

$$
\hat{\mathbf{b}}_{2}=\frac{\mathbf{r}^{m_{36} / m_{26}} \times \mathbf{r}^{m_{33} / m_{26}}}{\mid \mathbf{r}^{m_{36} / m_{26}} \times \mathbf{r}^{m_{33} / m_{26} \mid}}
$$

The heading vector of the rear frame is then $\hat{\mathbf{b}}_{1}=\hat{\mathbf{b}}_{2} \times \hat{\mathbf{n}}_{3}$ and $\hat{\mathbf{b}}_{3}=\hat{\mathbf{b}}_{1} \times \hat{\mathbf{b}}_{2}$ follows. These unit vectors define a reference frame that leans and yaws with the rear frame. We assumed that the rear frame pitch is negligible. The marker locations of the rider can now be expressed relative to the bicycle's inertial frame with reference to a point on the bicycle frame $m_{36}$. Equation (12) shows that the vector from any marker on the rider relative to $m_{36}$ can be expressed in the bicycle reference frame, $\mathbf{B}$, rather than the inertial frame, $\mathbf{N}$. This formulation was used in the PCA of the rider-only markers to look specifically at rider motion relative to the bicycle. The subscripts, $\mathbf{N}$ and $\mathbf{B}$, in (12) signify which reference frame the position vectors are expressed in.

$$
\mathbf{r}_{\mathbf{B}}^{m_{k} / m_{36}}=\left(\mathbf{r}_{\mathbf{N}}^{m_{k} / m_{36}} \cdot \hat{\mathbf{b}}_{1}\right) \hat{\mathbf{b}}_{1}+\left(\mathbf{r}_{\mathbf{N}}^{m_{k} / m_{36}} \cdot \hat{\mathbf{b}}_{2}\right) \hat{\mathbf{b}}_{2}+\left(\mathbf{r}_{\mathbf{N}}^{m_{k} / m_{36}} \cdot \hat{\mathbf{b}}_{3}\right) \hat{\mathbf{b}}_{3}
$$


A reference frame $\mathbf{D}$ that is aligned with the steering axis of the rear frame can be formulated by rotation about the $\hat{\mathbf{b}}_{2}$ axis through the steer axis angle $\lambda$, which is measured for each bicycle [9].

$$
\mathbf{D}=\left[\begin{array}{l}
\hat{\mathbf{d}}_{1} \\
\hat{\mathbf{d}}_{2} \\
\hat{\mathbf{d}}_{3}
\end{array}\right]=\left[\begin{array}{rr}
\cos \lambda 0 & -\sin \lambda \\
0 & 1 \\
\sin \lambda 0 & \cos \lambda
\end{array}\right]\left[\begin{array}{l}
\hat{\mathbf{b}}_{1} \\
\hat{\mathbf{b}}_{2} \\
\hat{\mathbf{b}}_{3}
\end{array}\right]
$$

The handlebar/fork inertial frame $\mathbf{E}$ is then calculated by defining $\hat{\mathbf{e}}_{2}$ to be aligned with the front wheel axle (14).

$$
\hat{\mathbf{e}}_{2}=\frac{\mathbf{r}^{m_{21} / n_{o}}-\mathbf{r}^{m_{27} / n_{o}}}{\left|\mathbf{r}^{m_{21} / n_{o}}-\mathbf{r}^{m_{27} / n_{o}}\right|}
$$

The handlebar/fork frame rotates around $\hat{\mathbf{d}}_{3}=\hat{\mathbf{e}}_{3}$ and then $\hat{\mathbf{e}}_{1}=\hat{\mathbf{e}}_{3} \times \hat{\mathbf{e}}_{2}$. Equation (15) gives the instantaneous rear wheel radius which is used to formulate the vector to the rear wheel contact point (16).

$$
\begin{gathered}
r_{\mathbf{R}}=-\frac{\mathbf{r}^{m_{36} / n_{o}} \cdot \hat{\mathbf{n}}_{3}}{\hat{\mathbf{b}}_{3} \cdot \hat{\mathbf{n}}_{3}} \\
\mathbf{r}^{m_{39} / n_{o}}=\mathbf{r}^{m_{36} / n_{o}}+r_{\mathbf{R}} \hat{\mathbf{b}}_{3}
\end{gathered}
$$

This now allows us to calculate six of the eight configuration variables of the bicycle as a function of time ( $q_{5}$ and $q_{8}$ are the rear and front wheel rotations, respectively).

Distance to the ground contact point: $q_{1}=\mathbf{r}^{m_{39} / n_{o}} \cdot \hat{\mathbf{n}}_{1}$,

Distance to the ground contact point: $q_{2}=\mathbf{r}^{m_{39} / n_{o}} \cdot \hat{\mathbf{n}}_{2}$,

$$
\begin{aligned}
& \text { Yaw angle: } q_{3}=\arccos \left(\hat{\mathbf{b}}_{1} \cdot \hat{\mathbf{n}}_{1}\right), \\
& \text { Roll angle: } q_{4}=\arccos \left(\hat{\mathbf{b}}_{3} \cdot \hat{\mathbf{n}}_{3}\right),
\end{aligned}
$$

$$
\text { Pitch angle: } q_{6}=0 \text {, }
$$

$$
\text { Steer angle: } q_{7}=\arccos \left(\hat{\mathbf{d}}_{1} \cdot \hat{\mathbf{e}}_{1}\right) \text {, }
$$

\section{References}

1. Meijaard, J.P., Papadopoulos, J.M., Ruina, A., Schwab, A.L.: Linearized dynamics equations for the balance and steer of a bicycle: a benchmark and review. Proc. R. Soc., A Math. Phys. Eng. Sci. 463(2084), 1955-1982 (2007)

2. Kooijman, J.D.G., Schwab, A.L., Meijaard, J.P.: Experimental validation of a model of an uncontrolled bicycle. Multibody Syst. Dyn. 19, 115-132 (2008)

3. Peterson, D.L., Hubbard, M.: Yaw rate and velocity tracking control of a hands-free bicycle. In: International Mechanical Engineering Congress and Exposition, Boston, Oct. 2008. ASME, New York (2008) 
4. Schwab, A.L., Kooijman, J.D.G., Meijaard, J.P.: Some recent developments in bicycle dynamics and control. In: Belyaev, A.K., Indeitsev, D.A. (eds.) Fourth European Conference on Structural Control (4ECSC), pp. 695-702. Institute of Problems in Mechanical Engineering, Russian Academy of Sciences, Moscow (2008)

5. Sharp, R.S.: On the stability and control of the bicycle. Appl. Mech. Rev. 61(6), 1-24 (2008)

6. Kooijman, J.D.G., Schwab, A.L., Moore, J.K.: Some observations on human control of a bicycle. In: Proceedings of the ASME 2009 International Design and Engineering Technical Conferences \& Computers and Information in Engineering Conference, 2009

7. Troje, N.F.: Decomposing biological motion: a framework for analysis and synthesis of human gait patterns. J. Vis. 2(5), 371-387 (2002)

8. Northern Digital Incorporated. Optotrak Certus Motion Capture System (2009)

9. Moore, J.K., Kooijman, J.D.G., Hubbard, M., Schwab, A.L.: A method for estimating physical properties of a combined bicycle and rider. In: Proceedings of the ASME 2009 International Design Engineering Technical Conferences \& Computers and Information in Engineering Conference, IDETC/CIE 2009, San Diego, CA, USA, August-September 2009. ASME, New York (2009)

10. Jolliffe, I.T.: Principal Component Analysis. Springer Series in Statistics, 2nd edn. Springer, New York (2002) 